\title{
Letters
}

Letters, which may not necessarily be published in full, should be restricted to not more than 250 words. When relevant, comment on the letter is sought from the author. Due to production schedules, it is normally not possible to publish letters received in response to material appearing in a particular issue earlier than the second or third subsequent issue.

\section{New drug comment - escitalopram}

Editor, - I refer to comments regarding escitalopram ('New drugs' Aust Prescr 2003;26:146-51). Only one study that investigated the efficacy of escitalopram in the treatment of major depression ${ }^{1}$ was cited in the article, when four other studies were available at the time of writing. ${ }^{2,3,4,5}$ The review concludes that escitalopram is a generic strategy.

Regulatory bodies advocate that companies must recognise the existence of chirality, that they should attempt to separate enantiomers, that the contribution of individual stereoisomers to the activity of interest should be assessed and that a rational decision regarding what stereoisomer to market should be made. ${ }^{6}$

The technology to separate the enantiomers of citalopram on a commercial scale has only recently been developed.

Preclinical studies have demonstrated that the antidepressant effect of citalopram resides primarily with the S-enantiomer. ${ }^{7}$ Escitalopram alone affects serotonin levels more effectively than escitalopram in combination with the R-enantiomer. ${ }^{8}$ The R-enantiomer decreases the association of the S-enantiomer with the human serotonin transporter via an allosteric mechanism. ${ }^{9}$ The R-enantiomer thus inhibits the active S-enantiomer.

A pooled analysis ${ }^{4}$ provided a sample size adequate for statistical comparisons between escitalopram and citalopram to be made. The results suggest that escitalopram may be superior to citalopram in terms of speed of onset and magnitude of clinical effects.

A meta-analysis ${ }^{10}$ has shown that escitalopram-treated patients have significantly higher response rates and an increased mean change from baseline in the Montgomery-

Asberg Depression Rating Scale (MADRS) at weeks one and eight when compared with citalopram. This superiority was more apparent with severely depressed patients.

Pharmacoeconomic analyses have found escitalopram has cost-effectiveness and cost-utility advantages over some other SSRIs and venlafaxine. ${ }^{11}$

Debbie Pelser

Medical Affairs Manager

Lundbeck Australia

Baulkham Hills, NSW

\section{References}

1. Burke WJ, Gergel I, Bose A. Fixed-dose trial of the single isomer SSRI escitalopram in depressed outpatients. J Clin Psychiatry 2002;63:331-6.
2. Wade A, Michael Lemming O, Bang Hedegaard K. Escitalopram $10 \mathrm{mg} /$ day is effective and well tolerated in a placebo-controlled study in depression in primary care. Int Clin Psychopharmacol 2002;17:95-102.

3. Lepola UM, Loft $\mathrm{H}$, Reines EH. Escitalopram (10-20 mg/day) is effective and well tolerated in a placebo-controlled study in depression in primary care. Int Clin Psychopharmacol 2003;18:211-7.

4. Gorman JM, Korotzer A, Su G. Efficacy comparison of escitalopram and citalopram in the treatment of major depressive disorder: pooled analysis of placebo-controlled trials. CNS Spectrums 2002;7(Suppl 1):40-4.

5. Montgomery SA, Loft H, Sanchez C, Reines EH, Papp M. Escitalopram (S-enantiomer of citalopram): clinical efficacy and onset of action predicted from a rat model. Pharmacol Toxicol 2001;88:282-6.

6. Caldwell J. Do single enantiomers have something special to offer? Hum Psychopharmacol Clin Exp 2001;16: S67-S71.

7. Hyttel J, Bogeso KP, Perregaard J, Sanchez C. The pharmacological effect of citalopram resides in the (S)-(+)-enantiomer. J Neural Transm (Gen Sect) 1992;88: 157-60.

8. Mork A, Kreilgaard M, Sanchez C. The R-enantiomer of citalopram counteracts escitalopram-induced increase in extracellular 5-HT in the frontal cortex of freely moving rats. Neuropharmacology 2003;45:167-73.

9. Wiborg O, Sanchez C. R-citalopram decreases the association of $\left[{ }^{3} \mathrm{H}\right]-\mathrm{S}$-citalopram with the human serotonin transporter by an allosteric mechanism [poster presented at the 16th Congress of the European College of Neuropsychopharmacology; 2003 September 20-24; Prague, Czech Republic]. Eur Coll Neuropsychopharmacol 2003;13(S4).

10. Auquier P, Robitail S, Llorca PM, Rive B. Comparison of escitalopram and citalopram efficacy: a meta-analysis. Int J Psychiatry Clin Pract 2003;7:259-68.

11. Croom KF, Plosker GL. Escitalopram: a pharmacoeconomic review of its use in depression. Pharmacoeconomics 2003;21:1185-209.

\section{Editorial comment:}

Lundbeck was invited to supply Australian Prescriber with information about escitalopram during the preparation of the new drug comment. The company informed the Editor that it would not release information because it was confidential. However, following publication of the new drug comment the company has supplied a dossier of data, including the previously confidential information. The Editorial Executive Committee welcomes the change of position by the company and hopes it will be an example other companies will follow. 
The additional information does not provide strong evidence that escitalopram has a clear clinical advantage over citalopram. Only two (references 3 and 5) of the four other studies included both drugs in humans. Reference 2 compares escitalopram with placebo, and reference 4 is a pooled analysis of other studies. Reference 5 was a combined study of patients and Polish rats, but it compared each drug with placebo rather than with each other. While reference 3 shows that significantly more patients were in remission after eight weeks of escitalopram than after citalopram $(52 \%$ versus $43 \%)$, neither treatment was significantly better than placebo. The mean change in the 60 point Montgomery-Asberg Depression Rating Scale (MADRS) was 15 points with escitalopram, 13.6 points with citalopram and 12 points with placebo.

It is not entirely clear which three studies were included in the pooled analysis (reference 4), but they are probably included in the company-sponsored meta-analysis (reference 10). The meta-analysis includes references 1, 3, an unpublished study and a conference abstract. While the unpublished study tended to favour citalopram, the metaanalysis showed a higher response rate with escitalopram (55.5\% versus $50.8 \%$ ). The mean change in the MADRS after eight weeks was approximately one point greater with escitalopram than with citalopram (1.02, confidence interval 0.09-1.95). None of the studies showed that patients responded significantly faster to escitalopram although the meta-analysis found an estimated difference of 0.63 (confidence interval 0.08-1.17) in the mean change in MADRS from week one of treatment. The clinical relevance of this difference is debatable.

The Pharmaceutical Benefits Advisory Committee has also concluded that the data do not demonstrate that escitalopram has superior efficacy to citalopram.

\section{New drug comment - pimecrolimus}

Editor, - The review of pimecrolimus (Elidel) in the 'New drugs' section (Aust Prescr 2003;26:146-51) states that children may be exposed to 'risks of immunosuppression'. Contrary to this view, clinical signs of systemic immunosuppression were not seen in the long-term paediatric studies. ${ }^{1,2}$ Some systemic adverse events were more common in the pimecrolimus group, but these were not significant when the time on study drug was taken into account. Pharmacokinetic studies demonstrated that blood concentrations of pimecrolimus following dermal application were below the limits of detection in the majority of paediatric patients, thus minimising the likelihood of a systemic effect.

Contrary to the review pimecrolimus did not enhance the carcinogenicity of UV light in animal models (see
TGA-approved product information). The lymphoma and thyroid adenomas mentioned by the reviewer were observed in animal studies only following oral administration of pimecrolimus, in which systemic exposure was much higher than that observed following clinical use of pimecrolimus cream. To date, clinical trial and postmarketing surveillance data do not indicate an increase in the risk of malignancy. Nevertheless Novartis recommends that patients avoid exposing skin areas treated with pimecrolimus to sunlight. Incorporating pimecrolimus into the management of atopic dermatitis was shown to improve long-term disease control and reduce the number of flares when compared with reactive use of topical corticosteroids. ${ }^{1,2}$ Pimecrolimus has not been associated with skin atrophy and is approved for use on all skin areas including the face, neck and intertriginous areas.

Natalie Jenkins

Senior Medical Information Associate

Victor Ferrari

Medical Adviser

Novartis Pharmaceuticals Australia

North Ryde, NSW

\section{References}

1. Kapp A, Papp K, Bingham A, Folster-Holst R, Ortonne JP, Potter PC, et al. Long-term management of atopic dermatitis in infants with topical pimecrolimus, a nonsteroid anti-inflammatory drug. J Allergy Clin Immunol 2002;110:277-84.

2. Wahn U, Bos JD, Goodfield M, Caputo R, Papp K, Manjra A, et al. Efficacy and safety of pimecrolimus cream in the long-term management of atopic dermatitis in children. Pediatrics 2002;110:e2.

\section{Editorial comment:}

It is reassuring to learn that postmarketing surveillance data have not yet shown evidence of malignancy in patients treated with pimecrolimus. However, unlike the Australian product information, the US product information does report an increase in the incidence of tumours in animals following dermal application of pimecrolimus.

While pimecrolimus may not enhance the carcinogenicity of UV light, the topical cream base enhances the development of skin tumours induced by UV radiation. Although the company advises patients to avoid sunlight, this may pose practical problems for patients applying pimecrolimus to the face or neck.

Although blood concentrations are low after topical application, absorption does occur. References 1 and 2 above did adjust for the duration of follow-up, but still showed a higher frequency of systemic symptoms, such as cough and fever, in children treated with pimecrolimus. 
Until more data are available, the Editorial Executive Committee still believes that pimecrolimus is not a first-line treatment and caution is needed, particularly when prescribing to infants. Neither the USA nor the UK have approved pimecrolimus for children under the age of two years.

\section{Oximetry}

Editor, - I enjoyed the review of oximetry by I. Young (Aust Prescr 2003;26:132-5), but was distressed to see the myth that 'nail polish must be removed' perpetuated in both the article and the self-test questions. An Australian woman can spend up to $\$ 1000$ each year on nail care and decoration. To have to remove that polish or enamel is both inconvenient and expensive.

A study of painted and unpainted nails, in the same people, tested 10 nail colours and found no significant differences in the $\mathrm{SpO}_{2}$ measured in the painted and unpainted fingers. ${ }^{1}$

It has been recommended that, since some nail polishes may reduce estimates of $\mathrm{SpO}_{2}$ by up to $6 \%$, the probe should be rotated through $90^{\circ}$ and mounted transversely in patients with nail polish or long nails. ${ }^{2}$ Personal observation in long endoscopy lists has shown no significant differences in saturations measured in the conventional way and measured transversely across the finger, in males or females.

We should abolish the myth of the necessity of nail polish removal once and for all and save nursing time.

John Paull

Consultant Anaesthetist

Launceston, Tas.

\section{References}

1. BrandTM, Brand ME, Jay GD. Enamel nail polish does not interfere with pulse oximetry among normoxic volunteers. J Clin Monit Comput 2002;17:93-6.

2. Ralston AC, Webb RK, Runciman WB. Potential errors in pulse oximetry. III: Effects of interferences, dyes, dyshaemoglobins and other pigments. Anaesthesia 1991;46:291-5.

Clinical Associate Professor I. Young, the author of the article, comments:

I was aware of the paper that suggests transverse mounting of the probe where nail polish is a problem and, of course, not all polish will cause a significant interference with the signal. My article does state that only strong superficial pigments are likely to be a problem.

I became aware of the Brand paper too late in the editorial process to change the article. The people in this study were normoxic individuals and some interference was found with strong blue and green pigments in vitro. However, I entirely accept Dr Paull's argument that it is not necessary to remove nail polish and regret that one of the questions attached to the article has erroneously emphasised this procedure.

\section{Traditional Chinese medicines}

Editor, -The authors of 'The quality and safety of traditional Chinese medicines' (Aust Prescr 2003;26:128-30) recommend the establishment of a quality testing system for Chinese herbs and their derivatives, in order to minimise mislabelling and identify undeclared components. This is based on the claims that the 'chemistry of herbal medicines is the foundation of their pharmacology', and that 'for most Chinese medicines the active components responsible for their pharmacological activities and clinical applications are not well defined'. The authors also point to the Chinese Medicine Registration Act in Victoria as an example of statutory regulation which will encourage the safe use of traditional Chinese medicines.

These recommendations are unexceptionable. However, these recommendations contain an irony, or a threat, depending on whether you subscribe to Western or Chinese medical systems. What is proposed is the application of Western scientific methods of analysis to Chinese medicines, in order to classify them as safe. In other words, the Chinese medical system, in order to survive in the dominant scientific culture, must subject itself to that culture's rules. This means that it cannot survive as a distinct and autonomous paradigm.

Mechanisms designed to ensure that Chinese medicines and practice (and any other traditional systems) continue to be recognised and respected, will ultimately ensure their demise.

Malcolm Parker

Associate Professor of Medical Ethics

School of Medicine

University of Queensland

Dr George Li, Dr Colin Duke and Professor Basil Roufogalis, the authors of the article, comment:

The letter raised the issue of the philosophy and position of complementary medicine. There are integrated and plural approaches. The article is in favour of an integrative approach. The recommendations are consistent with the recent report of the Expert Committee on Complementary Medicines. $^{1}$

We appreciate the concern about the possible overzealous application of modern scientific principles to the analysis of traditional medicines which have a different philosophical basis. In this article we addressed the issue of quality and safety of Chinese medicine, and we believe that the evaluation of safety of any device or therapeutic agent overrides cultural considerations. We agree that the assessment of safety of Chinese herbal medicines is complex, as they are made up 
of multiple components and used by practitioners in specific ways. Nevertheless, Chinese medicines contain chemical components that have specific biological actions requiring knowledge of the quality and nature of the ingredients.

The recommendations in our article do not address other aspects of Chinese medicine, whose principles can be maintained subject to appropriate evidence-based review. The recent report has recommended that governments introduce legislation to regulate practitioners of traditional Chinese medicine and dispensers of Chinese herbs. ${ }^{1}$ This development recognises the use and importance of traditional medicine systems in our society while requiring practitioners to be appropriately trained and accredited. The integrative approach to health care should aim to further develop the traditional system and bring it into the mainstream health care system. It should certainly not try to eliminate a traditional system that has been shown to be safe and effective.

\section{Reference}

1. Expert committee on complementary medicines in the health system. Complementary medicines in the Australian health system. Canberra: Commonwealth of Australia; 2003. http://www.tga.gov.au/docs/pdf/cmreport.pdf [cited 2004 March]

\section{BCG vaccination in Australia}

Editor, - In their articles on BCG vaccination (Aust Prescr 2003;26:144-6), neither Professor Simpson nor Air Vice-Marshal Short mentioned the potential for tuberculosis control offered by modern tuberculosis-specific tests that are unaffected by BCG vaccination. T-cell mediated immune responses to the tuberculosis-specific proteins ESAT-6 and CFP-10 (proteins not present in BCG or environmental mycobacteria) have been shown to be effective in diagnosing tuberculosis infection in BCG-vaccinated individuals. ${ }^{1,2}$

Unfortunately, despite intense interest in the literature, the use of tuberculosis-specific antigens in diagnostics has to date been limited by the complexity of the methodologies required to measure T-cell responses. Most methods require T-cell purification, counting, and culture, which are expensive and not suited for reproducible, robust diagnosis. However, the whole blood test, QuantiFERON-TB Gold, has now been released in Australia, after extensive testing overseas found it to have high specificity and sensitivity.

The Australian Defence Force may also note the US Centers for Disease Control endorses QuantiFERON-TB testing in the military. ${ }^{3}$ QuantiFERON testing detects significantly more active, infectious tuberculosis cases than Mantoux testing. ${ }^{4}$ The elimination of the confounding factors of $B C G$ vaccination and sensitisation to non-tuberculous mycobacteria makes the test an even more valuable diagnostic tool.

Tony Radford

Chief Executive Officer/Managing Director

Cellestis Ltd.

Melbourne

(Cellestis are manufacturers of QuantiFERON products)

\section{References}

1. Andersen P, Munk ME, Pollock JM, Doherty TM. Specific immune-based diagnosis of tuberculosis. Lancet 2000;356:1099-104.

2. Doherty TM, Demissie A, Olobo J, Wolday D, Britton S, EgualeT, et al. Immune responses to the Mycobacterium tuberculosis-specific antigen ESAT-6 signal subclinical infection among contacts of tuberculosis patients. J Clin Microbiol 2002;40:704-6.

3. Mazurek GH, Villarino ME. Guidelines for using the QuantiFERON(R)-TB test for diagnosing latent Mycobacterium tuberculosis infection. Centers for Disease Control and Prevention. MMWR 2003 Jan 31;52(RR-2):15-8.

4. Fietta A, Meloni F, Cascina A, Morosini M, Marena C, Troupioti P, et al. Comparison of a whole-blood interferon-gamma assay and tuberculin skin testing in patients with active tuberculosis and individuals at high or low risk of Mycobacterium tuberculosis infection. Am J Infect Control 2003;31:347-53.

Professor G. Simpson, the author of the article, comments: I did not discuss some of the newer tests for the diagnosis of latent tuberculous infection as the focus of the article was BCG. The tuberculin skin test (TST) or Mantoux test was mentioned as it is the test specified in current protocols concerned with BCG administration. Dr Radford is correct that the TST is imperfect and there is no doubt that sooner or later more sophisticated tests will replace it. Over the past 110 years however, it has proved remarkably robust and we do have vast amounts of data on outcomes related toTST results. These longitudinal data are not available for the newer tests.

The newer tests (QuantiFERON-TB Gold and ELISPOT) rely on detecting interferon gamma production by T-cells responding to antigens which are found in $\mathrm{M}$ tuberculosis, but not in BCG. This theoretically will enable us to remove the confounding effect of prior BCG vaccination from testing for subsequent tuberculosis infection. This is of course irrelevant in the context of pre-BCG vaccination testing. So far there is more published information on ELISPOT than QuantiFERONTB Gold. In the best study so far $^{1}$ in a tuberculosis outbreak in the UK the ELISPOT and Heaf test (a form ofTST) gave concordant results in $89 \%$ of those tested, suggesting that there is limited room for improved diagnostic accuracy with the newer tests. Nevertheless these are exciting 
developments in tuberculosis diagnosis and further studies including more longitudinal studies are likely to sound the death knell for the oldest diagnostic test in medicine.

\section{Reference}

1. Ewer K, Deeks J, Alvarez L, Bryant G, Waller S, Andersen $\mathrm{P}$, et al. Comparison of T-cell-based assay with tuberculin skin test for diagnosis of Mycobacterium tuberculosis infection in a school tuberculosis outbreak. Lancet 2003;361:1168-73.

Editor, - I noted with interest the photo illustrating the article 'BCG vaccine in Australia' (Aust Prescr 2003;26:144-6). What has happened to universal infection control precautions surely the person administering the injection should have been wearing gloves?

Anna McNulty

Director

Sydney Sexual Health Centre

\section{Drug-induced hyponatraemia}

Editor, -The useful review of drug-induced hyponatraemia (Aust Prescr 2003;26:114-7), states that 'blood glucose concentrations above $20 \mathrm{mmol} / \mathrm{L}$ can spuriously reduce the serum sodium concentration when measured by flame photometry'. This mistakenly implies that hyperglycaemia produces method-dependent pseudohyponatraemia of the type seen with marked hypertriglyceridaemia.

The hyponatraemia of marked hyperglycaemia is dilutional, from osmotic movement of water from the intracellular space, and is independent of method. ${ }^{1}$ The measured serum sodium concentration is analytically valid, but needs to be corrected before relating the value to the normal reference interval. $A$ useful correction, derived from a formula originally given in metric units, is to add a third to a half of the glucose excess in $\mathrm{mmol} / \mathrm{L}$ to the measured serum sodium concentration. ${ }^{2}$

Apparent hyponatraemia is a reassuring finding in severely hyperglycaemic dehydrated patients, as the serum sodium concentration, when corrected as above, is often close to normal. An apparently normal serum sodium concentration without correction implies hypernatraemia and indicates a water deficit much larger than the salt deficit. Severe hypernatraemia can then be anticipated during resuscitation with isotonic sodium chloride, especially if hyperglycaemia is rapidly corrected.

\section{J.R. Stockigt}

Professor of Medicine, Monash University

Department of Endocrinology and Diabetes

Alfred Hospital

Prahran, Vic.

\section{References}

1. Smith DM, McKenna K, Thompson CJ. Hyponatraemia. Clin Endocrinol 2000;52:667-78.

2. HillierTA, Abbott RD, Barrett EJ. Hyponatremia: evaluating the correction factor for hyperglycemia. Am J Med 1999;106:399-403.

Editor, - I found the article on hyponatraemia (Aust Prescr 2003;26:114-7) interesting. I have had several elderly patients who have developed severe hyponatraemia while on tramadol which has been corrected on its cessation.

Tramadol is thought to inhibit reuptake of serotonin which causes increased serotonin levels and presumably causes hyponatraemia due to a similar mechanism to the selective serotonin reuptake inhibitors. Tramadol was not included in Table 3 ‘Drugs commonly associated with hyponatraemia' and it is not listed in the product information as an adverse effect. I would appreciate the authors' comments.

Robin Hunter

Rehabilitation Physician

Brighton, Vic.

Dr S. Fourlanos and Dr P. Greenberg, the authors of the article, comment:

Professor Stockigt correctly indicates a factual error in our paper. We agree with him that the hyponatraemia associated with marked hyperglycaemia is dilutional, not method-dependent.

We note Dr Hunter's comment with interest. We were unaware of any association between tramadol and hyponatraemia, however the Adverse Drug Reactions Advisory Committee has received 12 reports.

\section{Bisphosphonates and avascular necrosis of the jaws}

Editor, - We wish to draw readers' attention to a potential drug-related cause of painful bone exposure in the maxilla complicating the healing of dental extractions. We have recently had four such cases in our unit all of whom were unresponsive to local medical and surgical treatments. All these patients were taking bisphosphonates; three were receiving pamidronate and one was receiving alendronate. This group of drugs act primarily through osteoclastic inhibition of bone resorption and are commonly prescribed in Australia to treat a variety of conditions including Paget's disease, hypercalcaemia of malignancy and osteoporosis.

On review of the literature we located a recent letter to the Journal of Oral and Maxillofacial Surgery which highlighted a growing epidemic of bisphosphonateinduced avascular necrosis of the jaws. ${ }^{1}$ The particular bisphosphonates implicated in the series of 36 patients are the potent nitrogen-containing bisphosphonates that are 
not metabolised, namely pamidronate and zoledronate. Interestingly, alendronate is also a potent nitrogen-containing bisphosphonate that is not metabolised. Consequently, we support the proposal of a link between avascular necrosis of the jaws and certain bisphosphonates currently prescribed in Australia. We draw this to the attention of practitioners prescribing these medications as a significant adverse effect.

\section{G.D. Carter}

Senior Registrar

A.N. Goss

Professor and Director

Oral and Maxillofacial Surgery Unit

Royal Adelaide Hospital, Adelaide Dental Hospital

Professor of Oral and Maxillofacial Surgery

School of Dentistry

University of Adelaide, Adelaide

\section{Reference}

1. Marx RE. Pamidronate (Aredia) and zoledronate (Zometa) induced avascular necrosis of the jaws: a growing epidemic. J Oral Maxillofac Surg 2003;61:1115-7.

\section{Promotion of over-the-counter medicines}

Editor, - I concur with Agnes Vitry ('And next: a flask of wine for Daddy?' Aust Prescr 2003;26:99-102). How is it possible that a drug company is allowed in Australia to promote drugs with free giveaways? I believe in most States pharmacists are not legally entitled to promote or advertise such medications.

The selection of an over-the-counter drug should be a therapeutic one, taking into consideration efficacy, adverse effects, safety, quality and quantity of drug information and cost. Free giveaways adversely influence such selections.

Although the evidence for many over-the-counter lines in Australia can be minimal, we nevertheless have a culture and tradition of usage. So even if evidence is scarce on the therapeutics of a drug, what little we have in addition to traditional usage, should play a far more important role than free giveaways.

Furthermore pharmacists should see this as another argument for drug companies to be allowed to promote such drugs in supermarkets, allowing unrestricted access to the public. For if the choice of a drug is dependent more on giveaways rather than therapeutics there is no reason to restrict its access to pharmacies. It is one thing to promote sunscreen with giveaways, it is quite another to do so with restricted over-the-counter products.

Derek Grubb

Pharmacist

Australind, WA

\section{Paediatric formulations}

Editor, -The editorial 'Why are children still therapeutic orphans?' (Aust Prescr 2003;26:122-6) rightly says '... even if a drug has good evidence of paediatric efficacy and safety, it may be unavailable in formulations ... that are suitable for children.'This may be true in developed countries, but in developing countries like India the situation is the other way round. We have many uncalled for paediatric formulations and combinations that probably do more harm than good.

Take for example the paediatric formulations and combinations of paracetamol, used for musculoskeletal disorders. There are:

- ten formulations of paracetamol (including syrup, suspension and dispersible tablets)

- three formulations containing paracetamol and ibuprofen

- one formulation containing paracetamol and ibuprofen with simethicone

- one formulation containing paracetamol and ibuprofen with magnesium trisilicate

- two formulations containing paracetamol and metoclopramide

- one formulation containing paracetamol and domperidone

- five formulations containing paracetamol and nimesulid.

In addition, there are many more paediatric formulations containing fixed dose combinations of paracetamol available to treat disorders of the respiratory system.

Wishvas Rane

Pune

India

\section{Withdrawal of useful drugs}

Editor, - What can be done when a well-known and frequently recommended drug just fades out of sight because the manufacturers won't make it any more? One's suspicions run riot, including that it is too cheap to make and to sell, and the profit margin is therefore too small. Maybe they want to sell something similar but more profitable. Patients' needs and the needs of the Australian community in general seem to be of no consequence!

Donald B. Reid

General practitioner

Bridgetown, WA 